\title{
Prevalence and Associated Factors of Soil-Transmitted Helminth Infections among Children Living with and without Open Defecation Practices in Northwest Ethiopia: A Comparative Cross- Sectional Study
}

\author{
Chalachew Muluneh, ${ }^{1 \star}$ Tadesse Hailu, ${ }^{2}$ and Getaneh Alemu ${ }^{2}$ \\ ${ }^{1}$ Medical Parasitology and Vector Control, Bahir Dar Zuria Woreda Health Office, Bahir Dar City, Ethiopia; ${ }^{2}$ Department of Medical Laboratory \\ Science, College of Medicine and Health Sciences, Bahir Dar University, Bahir Dar City, Ethiopia
}

\begin{abstract}
Soil-transmitted helminth (STH) infections cause devastating effect in human health. School-age children (SAC) account for the highest prevalence of STH infections in sub-Sahara. Open defecation practicing might be the major contributing factor, and creating an open defecation-free (ODF) declared community is also a big challenge. This study aimed to assess the prevalence of STH infections and associated factors among SAC in ODF declared and open defecation-practicing kebeles. A comparative cross-sectional study was conducted among 806 SAC from January 2019 to April 2019. Questionnaire-based data were collected using a structured questionnaire. Stool samples were collected and processed via the Kato-Katz technique. Prevalence and associated factors were computed with descriptive statistics and regression, respectively. Variables with a $P$-value $<0.05$ were considered as significantly associated. This study revealed that the prevalence of STH infections in open defecation-practicing and ODF declared kebeles were $39.0 \%$ and $30.0 \%$, respectively. Wearing open shoes, wearing shoes sometimes, and playing with soil were significantly associated $(P<0.01)$ with STH infections in ODF declared kebeles. Similarly, wearing shoes sometimes, not using latrine, and playing with soil were also significantly associated $(P<0.01)$ with STH infections in open defection-practicing kebeles. In conclusion, lower prevalence of STH infections was recorded in ODF declared than in open defection-practicing kebeles. Wearing shoes sometimes, playing with soil, and not using latrine were associated with STH infections. Therefore, creating an ODF environment and community awareness should be strengthened.
\end{abstract}

\section{INTRODUCTION}

Soil-transmitted helminths (STHs) are intestinal nematodes that are transmitted through contaminated hands, foods, soil, and water. The major STHs that infect humans include Ascaris lumbricoides, Trichuris trichiura, and hookworm species (Necator americanus and Ancylostoma duodenale). ${ }^{1}$ Globally, an estimated 4.5 billion individuals are at risk, $1-2$ billion individuals are infected, ${ }^{2}$ and more than five million disability-adjusted life years (DALYs) ${ }^{3}$ are lost because of STH infections. The greatest number of STH infections occurs in Latin America, tropical and subtropical regions of Asia, and sub-Saharan Africa. ${ }^{2,4}$

The high prevalence of STH infections in the developing countries is mainly due to open defecation practice, limited water source, and poor hygienic conditions, which are widespread problems of the rural community. ${ }^{5}$

School-age children (SAC) have the highest prevalence of STH infections worldwide. Around 400 million SAC are infected with STHs in the world. ${ }^{6}$ Soil-transmitted helminth infections are considered as the leading cause of sickness, school absenteeism, DALY lost, ${ }^{7}$ and physical and intellectual growth retardation, especially in SAC. ${ }^{8}$

Although the global target is to eliminate morbidity due to STH infections in children by $2020,{ }^{1}$ still, these infections cause huge health problems in countries like Ethiopia and affect millions of SAC. ${ }^{9}$ Improving water sanitation and hygiene (WASH), community mobilization and health education, integration of WASH, with mass drug administration (MDA) is still limited in many endemic areas.

In our study area, these control mechanisms are implemented especially improving WASH by using community-led

*Address correspondence to Chalachew Muluneh, Medical Parasitology and Vector Control, Bahir Dar City Health Office, Bahir Dar City, Ethiopia. E-mail: abenezerechale@gmail.com total sanitation and hygiene (CLTSH) strategies to create open defecation-free (ODF) declared environments in collaboration with Plan International Ethiopia and Federal Ministry of Health $(\mathrm{FMOH})$. Bahir Dar Zuria woreda heath office annual health management information system report indicated that 16 of the 32 kebeles of Bahir Dar Zuria district are ODF declared kebeles, whereas the rest are still open defecation-practicing (ODP) kebeles by the FMOH criterion. However, the role of creating ODF declared environments in reducing STH infection prevalence has not been assessed in the district so far. Besides, factors associated with STH infections in ODP and ODF declared areas are not well addressed in the study area. Therefore, this study aimed to compare the prevalence of STH infections in ODF declared and ODP kebeles as well as determine the factors associated with STH infections in Bahir Dar Zuria Woreda district.

\section{METHODS AND MATERIALS}

Study design, area, and period. A community-based comparative cross-sectional study was conducted among SAC in ODF declared and ODP kebeles of Bahir Dar Zuria district, northwest Ethiopia, from January 2019 to April 2019. The study area is located at 564 kilometers away from Addis Ababa. Bahir Dar Zuria district contains 32 rural kebeles, of which 16 kebeles have been announced and certified ODF during the years 2014-2016 by Bahir Dar Zuria Health Office in collaboration with Plan International Ethiopia and Organization for Rehabilitation and Development in Amhara.

Sample size determination. The sample size of the study was calculated using double population proportion based on findings of a study performed in rural Bahir Bar, northern Ethiopia (Hailu et al. ${ }^{13}$ ). Assuming the difference between ODF declared and ODP was $10 \%$, where the proportion of STH infections from ODF declared kebeles was $33 \%$ and the 
proportion of STH infections from ODP kebeles was $43 \%$. Using $95 \% \mathrm{Cl}, 80 \%$ power of test, and $10 \%$ contingency, the sample size was 403 for each group. The ratio between ODF declared and ODP kebeles was 1:1. Then, the total sample size was 806 .

Sample size per group $=P 1(1-P 1)+P 2(1-P 2) \times f(\alpha, \beta)$

$$
\begin{gathered}
(P 1-P 2)^{2} \\
=0.43(1-0.43)+0.33(1-0.33) \times 7.85=403 \\
(0.33-0.43)^{2}
\end{gathered}
$$

Total sample size $=806$ (we took 403 individuals in each group)

while $P 1=0.43, P 2=0.33$, and power of $80 \%-f(\alpha, \beta)=7.85$.

Sampling technique. A stratified sampling technique was used to stratify Bahir Dar Zuria district into ODF declared and ODP kebeles, and three kebeles were selected randomly in each stratum. Screening of households which have SAC and list 1-Nth using family folder at health posts (communitybased information system) was performed. The households with SAC were selected with a systematic random sampling technique. The study participants in each kebele were proportionally allocated based on the population of households which have SAC. A systematic random sampling technique was conducted to select the study participants or SAC. If there were two or more children in the same household, only one child was selected by the lottery method to participate in the study.

Data collection. A structured questionnaire was used to collect the sociodemographic data and environment- and behavior-related factors through face-to-face interviews. Following the questionnaire, approximately $2 \mathrm{~g}$ of fresh stool was collected using a stool cup. Each child was supplied with a plastic screw cup, applicator stick, and toilet paper, and was given appropriate instructions on how to bring fresh stool samples of their own. The stool samples were collected, labeled with a specific code number, and transported within 6 hours to Bahir Dar University Microbiology and Parasitology laboratory. Two Kato-Katz slides were prepared for each stool sample with $41.7 \mathrm{mg}$ of stool. ${ }^{10}$

In the Kato-Katz technique, the stool was pressed through a mesh screen to remove large particles. A portion of the sieved sample was transferred to the hole of a template on a slide. After filling the hole, the template was removed, and the remaining sample was covered with a piece of cellophane that was previously soaked in glycerol. The slides were examined, and the number of eggs was calculated per gram of feces. ${ }^{11}$

Quality control. All data collectors were trained before data collection, and the pretest was performed to validate the data collection instrument before conducting the study. During data collection, kebeles were coded and regular supervision was performed during the fieldwork. Each data collector checked the questionnaires for completeness before leaving each study participant's house. All filled questionnaires were reviewed at the end of the day by the supervisor. The reliability of the laboratory was assured by implementing quality control measures during the pre-analytical, analytical, and postanalytical steps. All materials, equipment, and procedures were adequately controlled. Each stool cup was properly labeled with their identification key. To minimize bias, each
Kato-Katz slide was examined by two laboratory personnel independently. From all of the slides, $10 \%$ was randomly selected and reexamined, and the discordant results were crosschecked by the principal investigator.

Statistical analysis. Data were entered and analyzed by SPSS version 21. The prevalence of STH infections was computed by descriptive statistics, whereas the associations of independent variables with the prevalence of STH infections were calculated by univariate logistic regression analysis. All variables with a $P$-value $<0.25$ in the univariate analysis were run in a multivariate logistic analysis at $95 \% \mathrm{Cl}$ to resolve the confounding effects. ${ }^{12} P<0.05$ was considered to be statistically significant.

Ethical consideration. The research proposal was ethically approved by the Ethical Review Committee of Bahir Dar University, College of Medicine and Health Sciences. Permission letters were obtained from Amhara Public Health Institute and Bahir Dar Zuria district Health Office. The purpose of the study and the entirely voluntary nature of the participants were clearly explained to the study participants/household heads before obtaining consent or assent. Study participants who tested positive for intestinal parasitic infections were communicated to the nearby health center and treated accordingly.

\section{RESULTS}

Sociodemographic characteristics of study participants. A total of 806 (403 in each ODF declared and ODP kebeles) children were recruited for this study. More than half $(54.3 \%)$ of the participant children were females. The mean, range, and SD of the study participants' age were 10.3, 6-14, and 2.29 years, respectively. The educational status of most children (89.9\%) was $0-4$ classes (92.8\% in ODF declared and $87.1 \%$ in ODP) (Table 1). All the study participants were rural inhabitants. Only one child was a Muslim religion follower, whereas all others were from an orthodox family.

Three hundred children, among 806 participants, were infected with at least one intestinal helminth $(37.2 \%$; $95 \% \mathrm{Cl}$ : 33.95-40.61). Five intestinal helminths were detected, of which the most frequently detected was hookworm (237/806), followed by $A$. lumbricoides (72/806). Schistosoma mansoni (20/806), Entrobius vermicularis (14/806), and Hymenolepis nana $(1 / 806)$ were the other helminths detected. The prevalence of helminth infections in ODF declared and ODP kebeles accounted for $32.8 \%$ (95\% Cl: 28.37-37.48) and 41.7\% (95\% Cl: 36.98-46.56), respectively. Soil-transmitted helminth infections accounted for $34.5 \%$ (95\% Cl: 31.29-37.84) prevalence among the whole $(n=806)$ study participants. The prevalence of STH infections in ODF declared and ODP Kebeles were 30.0\% (95\% Cl: 25.8-34.7) and 39.0\% (95\% Cl: 34.3-43.8), respectively, with high prevalence (34.5\%) in Yiloma kebele in ODF declared kebeles (Table 1).

The occurrence of STH infections in ODF declared kebeles was $31.2 \%$ in the $11-14$ age-group, $31.7 \%$ in females, and $30.2 \%$ in grade level 0-4 students. The increased prevalence of STH infections in ODP $(41.1 \%)$ was also recorded in the $6-10$ age-group, $45.3 \%$ in males, and $39 \%$ in children with $0-4$ grade level (Table 1).

From the total intestinal helminth infections, $A$. lumbricoides and hookworm parasites accounted for $34.5 \%$. Hookworm was the most common STH infections in both ODF declared $(27.5 \%)$ and ODP (31.3\%) kebeles. Single and double 
TABLE 1

Sociodemographic characteristics and distribution of soil-transmitted helminth infections prevalence of school-age children in selected ODF declared and ODP kebeles in Bahir Dar Zuria district, northwest Ethiopia, $2019(n=806)$

\begin{tabular}{|c|c|c|c|c|c|c|c|}
\hline \multirow[b]{2}{*}{ Variable } & \multirow[b]{2}{*}{ Category } & \multicolumn{2}{|c|}{ ODF declared } & \multicolumn{2}{|c|}{ ODP } & \multicolumn{2}{|c|}{ Total (ODF declared and ODP) } \\
\hline & & $N(\%)$ & Pos (\%) & $N(\%)$ & Pos (\%) & $N(\%)$ & Pos (\%) \\
\hline \multirow[t]{2}{*}{ Age-group (years) } & $6-10$ & $230(57.1)$ & $67(29.1)$ & $224(55.6)$ & $92(41.1)$ & 454 (56.3) & $159(35.0)$ \\
\hline & $11-14$ & 173 (42.9) & 54 (31.2) & $179(44.4)$ & 65 (36.3) & $352(43.7)$ & 119 (33.8) \\
\hline \multirow[t]{2}{*}{ Gender } & Male & $198(49.1)$ & $56(28.3)$ & $170(42.2)$ & 77 (45.3) & $368(45.7)$ & $133(36.1)$ \\
\hline & Female & 205 (50.9) & 65 (31.7) & 233 (57.8) & 80 (34.3) & 438 (54.3) & $145(33.1)$ \\
\hline \multirow[t]{2}{*}{ Children grade level } & $0-4$ & 374 (92.8) & $113(30.2)$ & $351(87.1)$ & $137(39.0)$ & 725 (89.9) & $250(34.5)$ \\
\hline & $5-8$ & $29(7.2)$ & 8 (27.6) & $52(12.9)$ & 20 (38.5) & $81(10.1)$ & 28 (34.6) \\
\hline \multirow[t]{3}{*}{ ODF kebele } & T/laguna & 119 (29.5) & 35 (29.4) & - & - & - & - \\
\hline & Chenta & $168(41.7)$ & $46(27.4)$ & - & - & - & - \\
\hline & Yiloma & $116(28.8)$ & $40(34.5)$ & - & - & - & - \\
\hline \multirow{3}{*}{ ODP kebele } & Debranta & - & - & $113(28.0)$ & $45(39.8)$ & - & - \\
\hline & Sekelet & - & - & 114 (28.3) & 45 (39.5) & - & - \\
\hline & Wonjeta & - & - & $176(43.7)$ & $67(38.1)$ & - & - \\
\hline Total & & $403(100)$ & $121(30.0)$ & 403 (100) & $157(39.0)$ & 806 (100) & 278 (34.5) \\
\hline
\end{tabular}

(hookworm $+A$. lumbricoides) infections in the whole study participants $(n=806)$ were found with infection rates of $30.6 \%$ and $3.9 \%$, respectively. Single infections were more common incidents among ODP kebeles children (34.5\%). Double infections were higher in ODP (4.5\%) kebeles than in ODF declared (3.2\%) kebeles (Table 2).

The intensity of STH infections. All STH infections were with light intensity in both ODF declared and ODP kebeles. The mean intensity of hookworm and $A$. lumbricoides was 205.6 and 122.1 eggs count per gram of stool, respectively, at ODF declared kebeles. In the same way, the mean intensity of STH infections of hookworm and $A$. lumbricoides at ODP kebeles was 300.9 and 142.5 eggs count per gram of stool, respectively.

Factors associated with STH infections in ODF kebeles. The multivariate logistic regression model revealed that children who wore open shoes were 2.30 times more exposed to STH infections than children who wore covered shoes (AOR = 230; 95\% Cl: 1.46-3.64). Likewise, children who wore their shoes sometimes were at 2.56 times higher risk of STH infections than children who always wore shoes (AOR $=2.56$; $95 \% \mathrm{Cl}: 1.25-5.25)$. In the same way, children playing with soil were 1.81 times more exposed to STH infections than children who could not play with soil $(\mathrm{AOR}=1.81,95 \% \mathrm{Cl}: 1.12-1.93)$ (Table 3).

Factors associated with STH infections in ODP kebeles. Multivariate logistic analysis on ODP kebeles also showed that children wearing shoes sometimes were 12.09 times more exposed than children who always wore their shoes (AOR = 12.09; 95\% Cl: 6.46-22.62). Similarly, children who were not using latrine had 2.15 times higher risk of STH infections than children who used the latrine $(\mathrm{AOR}=2.15 ; 95 \% \mathrm{Cl}: 1.20-3.84)$.
In the same way, children playing with soil were 4.62 times more exposed than children who did not play with soil (AOR = 4.62; 95\% Cl: 2.59-8.24). Children living in ODF declared kebeles had $49 \%$ decreased risk of infection with STHs (AOR $=0.51 ; 95 \% \mathrm{Cl}$ : 0.33-0.79) as compared with children living at ODP kebeles (Table 4).

\section{DISCUSSION}

Soil-transmitted helminth infections are the most common public health problem among SAC in developing countries. To minimize the impacts of STH infections, proper planning, implementation, and evaluation of intervention programs like ODF declared in endemic areas are very important. In the present study, the overall prevalence of STH infections was $34.5 \%$. This result was lower than the previous result obtained $(43.0 \%)$ in rural Bahir Dar, ${ }^{13}(69.2 \%)$ in rural Amhara region, ${ }^{14}$ and $(49.0 \%)$ in Jimma town. ${ }^{15}$ This result was also higher than the previous report obtained (12.6\%) in Ambo, west Ethiopia. ${ }^{16}$ This difference might be due to differences in diagnostic methods, environmental hygienic conditions, the difference in age-group, and WASH practice; for example, the study conducted in Jimma included 5-18 age-groups.

Proper utilization of latrine, good personal and environmental hygiene, and sanitation significantly decrease the prevalence of STHs in an institution or community. ${ }^{17}$ Implementation and application of ODF also decrease the prevalence of STH infections in a community by breaking the life cycle of STH infections. In the present study, the prevalence of STH infections in ODF declared kebeles (30\%) was low as compared with ODP (39.0\%) kebeles. A similar study

TABLE 2

Distribution of STHs identified from ODF and ODP kebeles children in Bahir Dar Zuria district, northwest Ethiopia, 2019

\begin{tabular}{lccc}
\hline \multicolumn{1}{c}{ Types of STHs } & $\begin{array}{c}\text { ODF kebeles infected } \\
\text { children, } N(\%)\end{array}$ & $\begin{array}{c}\text { ODP kebeles infected } \\
\text { children, } N(\%)\end{array}$ & Total, $N(\%)$ \\
\hline Hookworm & $111(27.5)$ & $126(31.3)$ & $237(29.4)$ \\
A.lumbricoides & $23(5.7)$ & $49(12.2)$ & $72(9.0)$ \\
Single infection & $108(26.8)$ & $139(34.5)$ & $247(30.6)$ \\
Double infection of hookworm & $13(3.2)$ & $18(4.5)$ & $31(3.9)$ \\
$\quad$ and $A$. lumbricoides & $121(30.0)$ & $157(38.9)$ & $278(34.5)$ \\
Total & & & \\
\hline
\end{tabular}

A. lumbricoides = Ascaris lumbricoides; ODF = open defecation-free; ODP = open defecation-practicing; $N$ = number examined; STH = soil-transmitted helminth . 
TABLE 3

Univariate and multivariate logistic regression analyses of factors associated with STH infections among school-age children in ODF declared kebeles in Bahir Dar Zuria district, northwest Ethiopia, $2019(n=403)$

\begin{tabular}{|c|c|c|c|c|c|c|c|}
\hline Variable & & $N$ & STH infections, $N(\%)$ & COR $(95 \% \mathrm{Cl})$ & $P$-value & AOR $(95 \% \mathrm{Cl})$ & $P$-value \\
\hline \multirow[t]{2}{*}{ Gender } & Male & 198 & $56(28.3)$ & $0.85(0.55-1.30)$ & 0.45 & - & - \\
\hline & Female & 205 & $65(31.7)$ & 1 & & & \\
\hline \multirow[t]{2}{*}{ Age-group (years) } & $6-10$ & 230 & 67 (29.1) & 1 & 0.65 & - & - \\
\hline & $11-14$ & 173 & 54 (31.2) & $1.10(0.72-1.70)$ & & & \\
\hline \multirow[t]{2}{*}{ Grade level of children } & $5-8$ & 29 & $8(27.6)$ & 1 & 0.76 & - & - \\
\hline & $0-4$ & 374 & $113(30.2)$ & $1.14(0.49-2.64)$ & & & \\
\hline \multirow[t]{2}{*}{ Family size } & $\geq 5$ & 235 & 79 (33.6) & 1.52 (0.98-2.36) & 0.06 & $1.39(0.87-2.21)$ & 0.17 \\
\hline & $<5$ & 168 & $42(25)$ & 1 & & 1 & \\
\hline \multirow[t]{2}{*}{ Latrine use } & No & 129 & 48 (37.2) & 1.63 (1.04-2.55 & $0.03^{\star}$ & $1.17(0.71-1.92)$ & 0.55 \\
\hline & Yes & 274 & 73 (26.6) & 1 & & 1 & \\
\hline \multirow[t]{2}{*}{ Types of shoes worn } & Open & 191 & $78(40.8)$ & $2.71(1.74-4.22)$ & $0.00^{\star}$ & $2.30(1.46-3.64)$ & $0.00^{*}$ \\
\hline & Covered & 212 & 43 (20.3) & 1 & & 1 & \\
\hline \multirow[t]{2}{*}{ Shoes worn } & Sometimes & 39 & $21(53.8)$ & 3.08 (1.58-6.02 & $0.00^{\star}$ & $2.56(1.25-5.25)$ & $0.01^{*}$ \\
\hline & Always & 364 & $100(27.5)$ & 1 & & 1 & \\
\hline \multirow[t]{2}{*}{ Drinking water source } & Tap water & 221 & $58(26.2)$ & 1 & 0.07 & 1 & 0.10 \\
\hline & Others & 182 & $63(34.6)$ & 1.49 (0.97-2.28) & & $1.48(0.93-2.37)$ & \\
\hline \multirow[t]{2}{*}{ Handwashing before meal } & No & 4 & $2(50)$ & $2.35(0.33-16.90)$ & 0.39 & - & - \\
\hline & Yes & 399 & $119(29.8)$ & 1 & & & \\
\hline \multirow[t]{2}{*}{ Washing vegetable } & No & 103 & $33(32.0)$ & $1.14(0.70-1.84)$ & 0.60 & & - \\
\hline & Yes & 300 & 88 (29.3) & 1 & & & \\
\hline \multirow[t]{2}{*}{ Playing with soil } & No & 192 & 44 (22.9) & 1 & $0.03^{\star}$ & 1 & $0.02^{*}$ \\
\hline & Yes & 211 & 77 (36.5) & 1.93 (1.25-2.99) & & 1.81(1.12-1.93) & \\
\hline \multirow[t]{2}{*}{ Nail trimming } & Not trimmed & 131 & 49 (37.4) & 1.66 (1.06-8.68) & $0.03^{\star}$ & $1.18(0.72-1.93)$ & 0.52 \\
\hline & Trimmed & 272 & $72(26.5)$ & 1 & & 1 & \\
\hline
\end{tabular}
${ }^{*} P<0.05$.

conducted in India showed the prevalence of worm infection was higher (44\%) in the ODP villages than (36\%) in the ODF declared villages. ${ }^{18}$ The present result was also supported by a study carried out in Coted'Ivoire that showed the successful implementation of CLTSH in a community decreases the STH infection prevalence. ${ }^{19}$ The present low prevalence of STH infections in ODF declared kebeles was supported by studies conducted in governmental and private schools in Jimma ${ }^{20}$ and Nigeria. ${ }^{21}$ In these studies, the sanitation and hygiene practice is better in private schools which showed a low prevalence of STH infections. Generally, this study justified as the prevalence of STH infections might be highly dependent

TABLE 4

Univariate and multivariate logistic regression analyses of factors associated with STH infections among school-age children in ODP kebeles in Bahir Dar Zuria district, northwest Ethiopia, 2019

\begin{tabular}{|c|c|c|c|c|c|c|c|}
\hline Variable & & $N$ & STH infections, $N(\%)$ & $\operatorname{COR}(95 \% \mathrm{Cl})$ & $P$-value & AOR (95\% Cl) & $P$-value \\
\hline \multirow[t]{2}{*}{ Gender } & Male & 170 & $77(45.3)$ & $1.58(1.06-2.38)$ & $0.03^{*}$ & $1.01(0.56-1.83)$ & 0.96 \\
\hline & Female & 233 & $80(34.3)$ & 1 & & 1 & \\
\hline \multirow[t]{2}{*}{ Age-group (years) } & $6-10$ & 224 & 92 (41.1) & 1 & 0.33 & & \\
\hline & $11-14$ & 179 & 65 (36.3) & $0.82(0.55-1.23)$ & & & \\
\hline \multirow[t]{2}{*}{ Grade level of children } & $5-8$ & 52 & $20(38.5)$ & 1 & 0.98 & & \\
\hline & $0-4$ & 351 & 137 (39.0) & $0.98(0.54-1.78$ & & & \\
\hline \multirow[t]{2}{*}{ Family size } & $\geq 5$ & 231 & 90 (38.9) & $1.00(0.67-1.50)$ & 0.99 & & \\
\hline & $<5$ & 172 & 67 (38.9) & 1 & & & \\
\hline \multirow[t]{2}{*}{ Latrine use } & No & 225 & 116 (51.6) & $3.56(2.30-5.50)$ & $0.00^{\star}$ & $2.15(1.20-3.84)$ & $0.01^{*}$ \\
\hline & Yes & 178 & $41(23.0)$ & 1 & & 1 & \\
\hline \multirow[t]{2}{*}{ Types of shoes worn } & Open & 243 & 115 (47.3) & $2.52(1.64-3.89)$ & $0.00^{\star}$ & $0.83(0.43-1.61)$ & 0.59 \\
\hline & Covered & 160 & $42(26.3)$ & 1 & & 1 & \\
\hline \multirow{2}{*}{ Shoes worn } & Sometimes & 202 & 137 (67.8) & $19.08(11.03-32.99)$ & $0.00^{\star}$ & 12.09 (6.46-22.62) & $0.00^{\star}$ \\
\hline & Always & 201 & $20(9.9)$ & 1 & & 1 & \\
\hline \multirow[t]{2}{*}{ Drinking water source } & Tap water & 170 & 53 (31.2) & 1 & $0.01^{\star}$ & $1.00(0.56-1.79)$ & 0.99 \\
\hline & Others & 233 & 104 (44.6) & $1.78(1.18-2.69)$ & & 1 & \\
\hline \multirow[t]{2}{*}{ Handwashing before meal } & No & 5 & $3(60)$ & $2.38(0.39-14.39)$ & 0.35 & & \\
\hline & Yes & 8 & $154(38.7)$ & 1 & & & \\
\hline \multirow[t]{2}{*}{ Washing vegetable } & No & 89 & 53 (59.6) & 2.97 (1.83-4.82) & $0.00^{*}$ & $1.69(0.86-3.32)$ & 0.13 \\
\hline & Yes & 314 & $104(33.1)$ & 1 & & 1 & \\
\hline \multirow[t]{2}{*}{ Playing with soil } & No & 211 & $34(16.1)$ & 1 & $0.00^{*}$ & 1 & $0.00^{\star}$ \\
\hline & Yes & 192 & $123(64.1)$ & $9.28(5.80-14.86$ & & $4.62(2.59-8.24)$ & \\
\hline \multirow[t]{2}{*}{ Nail trimming } & Not trimmed & 165 & 94 (56.9) & $3.68(0.83-2.13)$ & $0.00^{*}$ & $1.61(0.90-2.86)$ & 0.11 \\
\hline & Trimmed & 238 & 63 (26.5) & 1 & & 1 & \\
\hline \multirow[t]{2}{*}{ Group } & ODP & 403 & 157 (38.9) & 1.49 (1.11-1.99) & $0.01^{*}$ & $0.51(0.33-0.79)$ & 0.00 \\
\hline & ODF & 403 & $121(30.0)$ & 1 & & 1 & \\
\hline
\end{tabular}

ODF = open defecation-free; ODP = open defecation-practicing; STH = soil-transmitted helminth, $N$ = number examined.

${ }^{\star} N=$ number examined. 

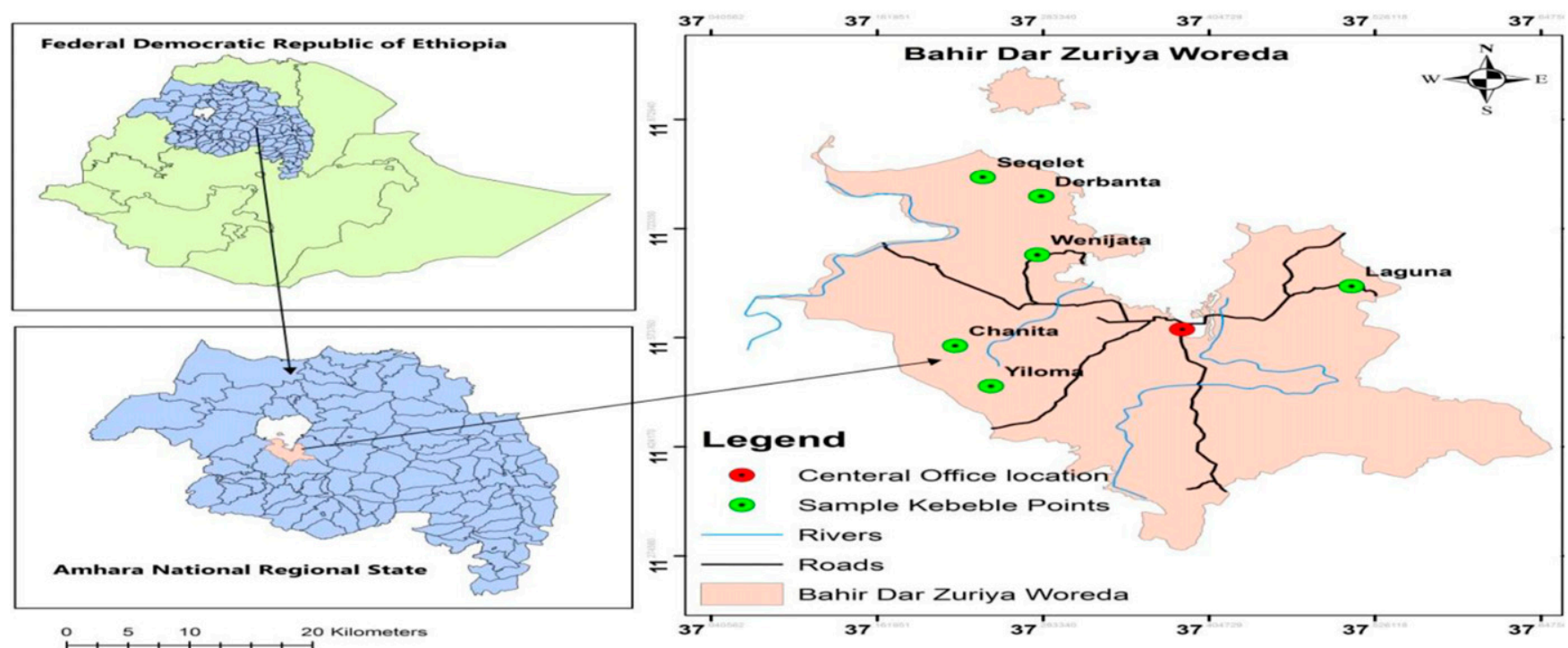

FIGURE 1. Map of the Bahir Dar Zuria woreda and the study sites. This figure appears in color at www.ajtmh.org.

on the sanitation practice taking place at the individual, institutional, and community levels.

Children are highly at risk for hookworm infections. They acquire hookworm infections when they walk barefoot and play with soil which contains the infective stages (larvae). Similarly, children get $A$. lumbricoides infections through the ingestion of contaminated food, water, and soil, because the soil is contaminated with ova of $A$. lumbricoides and

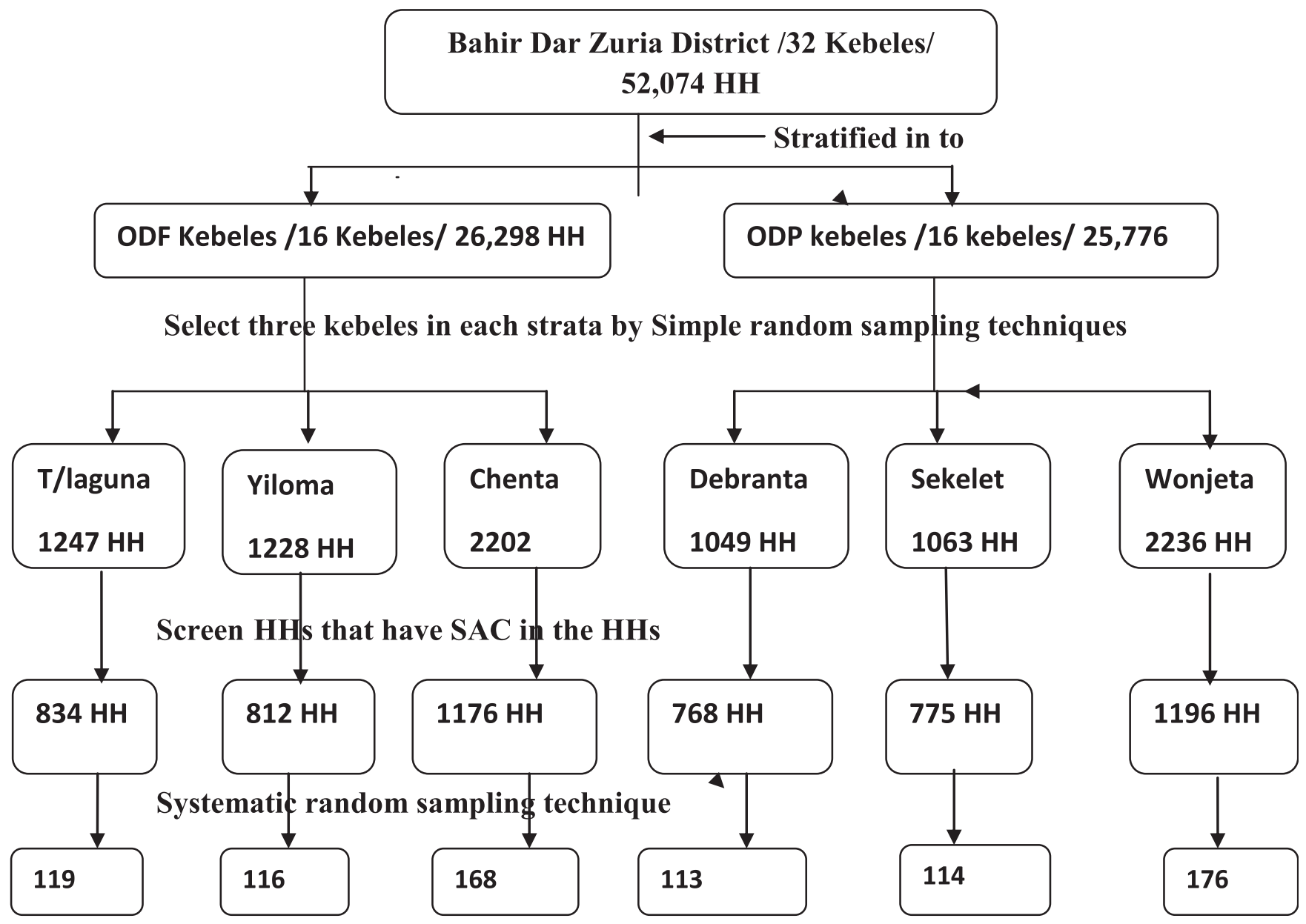

FIGURE 2. Participant flowchart showing participant selection. ${ }^{*} \mathrm{FF}=$ family folder; $\mathrm{HP}=$ health post; $\mathrm{HH}=$ household; $N=$ total number of selected households; $K=$ interval $\mathrm{b} / \mathrm{n}$ households. 
hookworm parasites through open defecation. Creating the ODF environment helps to prevent contamination of soil with hookworm larvae and decrease the prevalence. In the present study, hookworm infections were the commonest in children living in ODF declared (27.3\%) and ODP (31.6\%) kebeles, and the prevalence decreases in ODF declared kebeles. This result was consistent with the previous finding conducted among individuals in ODF declared (16.3\%) and ODP (20.9\%) villages in India. ${ }^{18}$

Hookworm infections remain common throughout much of sub-Saharan Africa, ranging from 2.3\% in Eritrea to $30.5 \%$ in the Central African Republic. ${ }^{22}$ In the present study, hookworm was the most frequent STH infections (29.4\%). This result was consistent with the previous findings performed in rural Bahir Dar, ${ }^{13}$ and in rural highlands of northwest Ethiopia ${ }^{14}$ and India, ${ }^{23}$ but higher than the previous result obtained in Ambo town in western Ethiopia. ${ }^{16}$ The difference might be due to the difference in diagnostic method, sample size, and geographical location.

Ascaris lumbricoides is the predominant STH infection globally. ${ }^{22}$ The prevalence of $A$. lumbricoides infections among the study participants in the present study was $8.9 \%$. This result was consistent with findings of $8.5 \%$ and $7.8 \%$ obtained in rural highlands of northwest Ethiopia ${ }^{14}$ and Ambo town, western Ethiopia, ${ }^{16}$ respectively. However, it was lower than previous records that obtained $23.4 \%$ and $52 \%$ in Jimma town ${ }^{15}$ and Bahrin state of India, ${ }^{23}$ respectively. The difference might be due to the low levels of sanitation facilities and practice. For example, the study conducted in India showed that only $17 \%$ of the participants had a toilet.

The intensity of parasitic infections is very crucial for parasite infectivity and transmission. In the present study, the mean intensity of hookworm and $A$. lumbricoides infections were 205.6 and 122.1 at ODF declared kebeles, respectively. Similarly, the mean intensity of hookworm and $A$. lumbricoides infections were 300.9 and 142.5 at ODP kebeles, respectively. This result was lower than the previous study carried out in India, 1803 for $A$. lumbricoides and 416 for hookworm. ${ }^{24}$ This difference might be due to different environmental hygienic conditions, sample size, deworming, and difference in the data collection time. According to the WHO classification, in the current study, only light infections were observed in all positive SAC; it might be due to the administration of MDA every 6 months in the study area. This result was similar to the study carried out in Ambo town. ${ }^{16}$

In the current study, the odds of children who wore open shoes were 2.30 times more exposed to STH infections than children who wore covered shoes $(\mathrm{AOR}=2.30 ; 95 \% \mathrm{Cl}$ : 1.46-3.64). The possible justification might be due to the absence of full protection of soil contamination with open shoes as covered shoes. In the same way, children who wear shoes sometimes were 2.56 times at a higher risk of STH infections than children who wore shoes always $(\mathrm{AOR}=2.56,95 \% \mathrm{Cl}$ : 1.25-5.25). This result was consistent with the previous study conducted in Durebet town, northwest Ethiopia. ${ }^{25}$ Similar to the present study, previous studies have shown that wearing shoes reduces hookworm infection by $71 \%{ }^{17}$ and is 2.1 times more protective for STH infections. ${ }^{26}$

Absence of latrine utilization $(P=0.01)$, occasionally/not wearing shoes $(P=0.00)$, and playing with soil $(P=0.00)$ were associated factors for STH infections in ODP kebeles. The proper utilization of latrine decreases the prevalence of STH infections. In the present study, children not using latrine were 2.15 times more exposed to STH infections than children using latrine in ODP kebeles (AOR $=2.15$; 95\% Cl:1.20-3.84). A similar study showed that proper latrine use decreased STH infections. ${ }^{17,27,28}$ This can be justified that awareness of STH infections and effective utilization of latrine are the target of creating ODF which leads to a decrease in environmental contamination with human feces.

Our environment is contaminated with the ova of STHs because of open defecation. As a result, children contaminated their hands when they play with soil. ${ }^{29}$ In the present study, children playing with soil were 4.62 times more exposed to STH infections than children who did not play with soil $(\mathrm{AOR}=4.62 ; 95 \% \mathrm{Cl}$ : 2.59-8.24). The possible justification might be that children living in our study area played with soil and mud, drilled holes in the soil, and played volleyball and football, which lead to parasitic infections.

The limitation of this study was using a single stool sample that has a low chance of getting the parasites in the Kato-Katz technique and unable to determine the contamination rate of soil in the ODF declared and ODP kebeles.

\section{CONCLUSION AND RECOMMENDATION}

The prevalence of STH infections was lower among children living in ODF declared kebeles than in ODP kebeles. All STH infections were with light intensity in both ODP and ODF declared kebeles. Open shoe wearing, wearing shoes sometimes, and playing with soil were associated with STH infections in ODF kebeles, and wearing shoes sometimes, playing with soil, and not using latrine were associated with STH infections in ODP kebeles. Therefore, upgrading ODF strategies in ODP kebeles, coupled with close follow-up and monitoring, sustains ODF environment free of STHs; proper awareness creations aiming to change the knowledge, attitude, and practice of the community should be strengthened in the study area.

Received September 23, 2019. Accepted for publication April 2, 2020. Published online May 11, 2020.

Acknowledgments: We acknowledge Bahir Dar University College of Medicine and Health Sciences School of Health Sciences, Department of Medical Laboratory Science for its contributory role on academic issues, financial support, and allowing us to use all the available resources of the university. The American Society of Tropical Medicine and Hygiene (ASTMH) assisted with publication expenses.

Financial support: The source of budget for the project was provided by Bahir Dar University.

Authors' addresses: Chalachew Muluneh, Medical Parasitology and Vector Control, Bahir Dar City Health Office, Bahir Dar City, Ethiopia, E-mail: abenezerechale@gmail.com. Tadesse Hailu and Getaneh Alemu, Department of Medical Laboratory Science, College of Medicine and Health Sciences, Bahir Dar University, Bahir Dar City, Ethiopia, E-mails: tadessehailu89@gmail.com and getanehmlt@ gmail.com.

\section{REFERENCES}

1. World health organization, 2018. Soil Transmitted Helmenths. Available at: https://www.who.int/intestinal_worms/. Accessed December 22, 2018.

2. Tefera E, Belay T, Mekonnen SK, Zeynudin A, Belachew T, 2017. Prevalence and intensity of soil transmitted helminths among school children of mendera elementary school, Jimma, southwest Ethiopia. Pan Afr Med J 27: 88. 
3. Murray CJ et al., 2012. Disability-adjusted life years (DALYs) for 291 diseases and injuries in 21 regions, 1990-2010: a systematic analysis for the Global Burden of Disease Study 2010. Lancet 380: 2197-2223.

4. World Health Organization, 2019. Soil-Transmitted Helminth Infections. Available at: https://www.who.int/news-room/factsheets. Accessed September 24, 2019.

5. Ayalew AM, Mekonnen WT, Abaya SW, Mekonnen ZA, 2018. Assessment of diarrhea and its associated factors in under-five children among open defecation and open defecation-free rural settings of Dangla district, northwest Ethiopia. J Environ Public Health 2018: 4271915.

6. Abossie A, Seid M, 2011. Assessment of the prevalence of intestinal parasitosis and associated risk factors among primary school children in Chencha town, Southern Ethiopia. BMC Public Health 14: 166.

7. World Health Organization, 2011. Helminth Control in School-Age Children: A Guide for Managers of Control Programmes, 2nd edition. Geneva, Switzerland: WHO. Available at: https:// www.who.int/neglected_diseases/resources/9789241548267/en/. Accessed May 12, 2019.

8. Bethony J, Brooker S, Albonico M, Geiger SM, Loukas A, Diemert D, Hotez PJ, 2006. Soil-transmitted helminth infections: ascariasis, trichuriasis, and hookworm. Lancet 367: 1521-1532.

9. Negussu N, Mengistu B, Kebede B, Deribe K, Ejigu E, Tadesse G, Mekete K, Sileshi M, 2017. Ethiopia schistosomiasis and soiltransmitted helminthes control programme: progress and prospects. Ethiop Med J 55 (Suppl 1): 75-80.

10. Williams JE, 1992. Basic laboratory methods in medical parasitology: Geneva: World Health Organization, 1991. viii+114 pp. Price Sw. fr. 21.00, US\$18. 90 (in developing countries Sw. fr.14.70). ISBN 92-4-1544104. Trans $R$ Soc Trop Med Hyg 86: 701.

11. Montresor A, Crompton DWT, Hall A, Bundy DAP, Savioli L, 1998. Guidelines for the Evaluation of Soil-Transmitted Helminthiasis and Schistosomiasis at Community Level. Geneva, Switzerland: Schistomiasis and Intestinal Parasites Unit, Division of Tropical Diseases. Available at: https://apps.who.int/iris/handle/ 10665/63821. Accessed December 25, 2019.

12. Deribe K, Meribo K, Gebre T, Hailu A, Ali A, Aseffa A, Davey G, 2012. The burden of neglected tropical diseases in Ethiopia, and opportunities for integrated control and elimination. Parasit Vectors 5: 240.

13. Hailu T, Abera B, Mulu W, Alemu M, Yizengaw E, Genanew A, 2018. Efficacy of single dose albendazole and praziquantel drugs among helminth-infected school children at rural Bahir Dar, northwest Ethiopia. Trop Doct 48: 270-272.

14. Amor A et al., 2016. High prevalence of strongyloides stercoralis in school-aged children in a rural highland of north-western Ethiopia: the role of intensive diagnostic work-up. Parasit Vectors 9: 617.

15. Mekonnen Z, Getachew M, Bogers J, Vercruysse J, Levecke B, 2019. Assessment of seasonality in soil-transmitted helminth infections across 14 schools in Jimma town, Ethiopia. Pan Afr Med J 32: 6.
16. Samuel F, Demsew A, Alem Y, Hailesilassie Y, 2017. Soil transmitted helminthiasis and associated risk factors among elementary school children in ambo town, western Ethiopia. BMC Public Health 17: 791.

17. Strunz EC, Addiss DG, Stocks ME, Ogden S, Utzinger J, Freeman MC, 2014. Water, sanitation, hygiene, and soil-transmitted helminth infection: a systematic review and meta-analysis. PLoS Med 11: e1001620.

18. Chakma T, Godfrey S, Bhatt J, Rao PV, Meshram P, Kinyanjui SS, 2008. Cross- sectional health indicator study of open defecation-free villages in Madhya Pradesh, India. Waterlines 27: 236-247.

19. Hürlimann $E$ et al., 2018. Effect of an integrated intervention package of preventive chemotherapy, community-led total sanitation and health education on the prevalence of helminth and intestinal protozoa infections in Côte d'Ivoire. Parasit Vectors 11: 115

20. Debalke S, Worku A, Jahur N, Mekonnen Z, 2013. Soil transmitted helminths and associated factors among schoolchildren in government and private primary school in Jimma town, southwest Ethiopia. Ethiop J Health Sci 23: 237-244.

21. Esiet ULP, Edet IA, 2017. Comparative prevalence of intestinal parasites among children in public and private schools in Calabar South, Calabar, Cross River state, Nigeria. Am J Res Commun 5: 80-97.

22. Pullan RL, Smith JL, Jasrasaria R, Brooker SJ, 2014. Global numbers of infection and disease burden of soil transmitted helminths infections in 2010. Parasit Vectors 7: 37.

23. Greenland $\mathrm{K}$ et al., 2015. The epidemiology of soil-transmitted helminths in Bihar state, India. PLoS Neg/ Trop Dis 9: e0003790.

24. Ganguly $S$ et al., 2017. High prevalence of soil-transmitted helminth infections among primary school children, Uttar Pradesh, India, 2015. Infect Dis Poverty 6: 139.

25. Alelign T, Degarege A, Erko B, 2015. Soil-transmitted helminth infections and associated risk factors among schoolchildren in Durbete town, northwestern Ethiopia. J Parasitol Res 2015: 641602.

26. Muslim A, Sofian SM, Shaari SA, Hoh BP, Lim YAL, 2019. Prevalence, intensity and associated risk factors of soil transmitted helminth infections: a comparison between Negritos (indigenous) in inland jungle and those in resettlement at town peripheries. PLoS Negl Trop Dis 13: e0007331.

27. Yimam YT, Gelaye KA, Chercos DH, 2014. Latrine utilization and associated factors among people living in rural areas of Denbia district, northwest Ethiopia, 2013, a cross-sectional study. Pan Afr Med J 18: 334

28. Ziegelbauer K, Speich B, Mäusezahl D, Bos R, Keiser J, Utzinger $J$, 2012. Effect of sanitation on soil-transmitted helminths infection: systematic review and meta-analysis. PLoS Med 9: e1001162.

29. Hassan AA, Oyebamiji DA, Idowu OF, 2017. Spatial patterns of soil-transmitted helminths in soil environment around Ibadan, an endemic area in south-west Nigeria. Niger $J$ Parasitol 38: 179-184. 